THES IS

- -

ACOUSTIC

SCATTERING BY

FIUID SPHERES

Submitted by

Harlan G. Frey

In partial fulfillment of the requirements

for the Degree of Master of Sclence

in Physics

Colorado State University

Fort Coll1ns, Colorado

May, 1962

LIGRARY

COLORADO STATE UNIVERSITY

FORT COLLINS, COLORADO 
COLORADO STATE UNIVERSITY

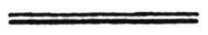

$$
\text { March } 1962
$$

WE HEREBY RECOMMEND THAT THE THESIS PREPARED UNDER OUR

SUPERVISION BY HARLAN GLEN FREY

ENTITLED ACOUSTIC SCATTERING BY FIUID SPHERES

BE ACCEPTED AS FULFILLING THIS PART OF THE REQUIREMENTS FOR THE DEGREE OF MASTER OF SCIENCE.

Committee on Graduate Work

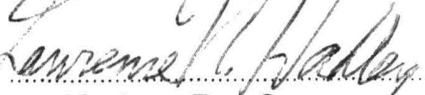

Major Professor
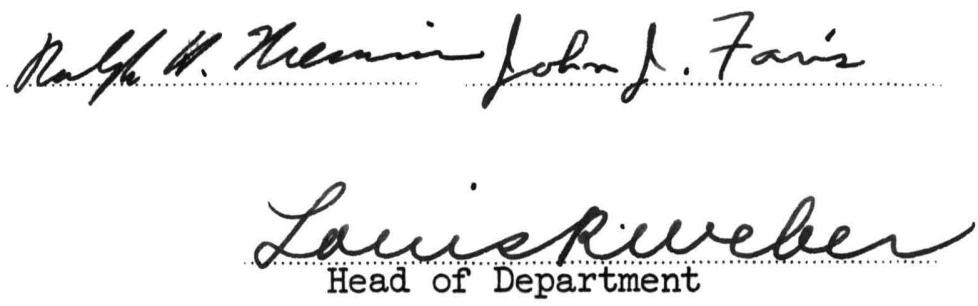

Examination Satisfactory

Committee on Final Examination
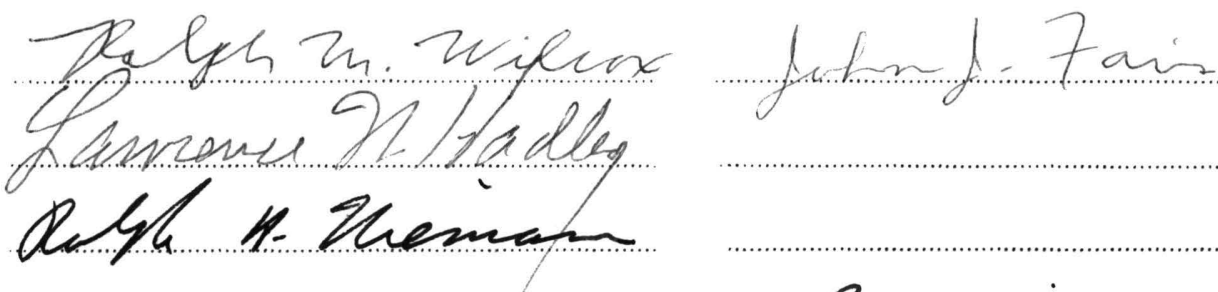

Permission to publish this report or any part of it must be obtained from the Dean of the Graduate School. 


\section{ACKNOWTED GEMENTS}

The author vishes to express his sincere Indebtedness to Dr. RaIph R. Goodman, for constant advice and assistance during this study and through whose efforts the support of the offlce of Naval Research Contract Nonr 1610(05) was obtained. The support of this contract is gratefully acknowledged. Appreciation is extended to Dr. Louls $R$. Weber, Dr. Iawrence $N$. Hadley and Dr. Ralph H. N1emann for their service on the graduate committee. 
TABIE OF CONTENTS

Page No.

OHAPTER I:

Introduction . . . . . 1

CHAPTER II:

General Solutions for Sperical

Scattering . . . . 4

CHAPTER III:

$\begin{array}{ll}\text { An Approximation Method for } & \\ \text { Scattering by a Sphere } & \\ \end{array}$

CHAPTER IV:

The Back-Scattering of Pulses

Prom a Fluid Sphere . . . $\quad 21$

CHAPTER V:

Summary and D1scussion - . 34

APPENDIX A:

Evaluation of Infinite Sum . - 38

APPENDIX B:

Fourler Transforms and Contours

of Integration . . . .

41

APPENDIX C:

The Born Approximation for

SphericaI Scattering . . 45

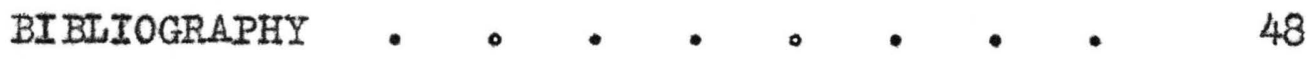




\section{CHAPTER I}

\section{INTRODUCT ION}

Throughout physics a great variety of scattering problems is encountered. In classical physics, laws have been formulated for scattering by both microscopic and macroscopic objects. For example, Rutherford formulated a scattering law to describe the scattering of charged particles by nucle1. In the field of quantum mechanios, the scattering of electrons from atoms is st1ll of considerable interest. In the last half century the scattering of electromagnetic energy has been extensively studled, and in the last twenty years has become increasingly important due to advances in radar technology. Beginning with the investigations of Iord Rayleigh in the 19th century, the fleld of acoustical scattering has also become increasingly important. The amount of research being reported in recent issues of acoustical journals indicates the extent of interest in scattering theory.

Upon 1nvestigation one is struck by the similarity in the methods of approach to scattering problems in quantum mechanics, nuclear physics, sound and electromagnetic theory. Thus, a contribution in one field generally extends the knowledge in all of them. This thesis is concerned with a problem in acoustic 
scattering theory and w1II be confined to that field. Specifically, it is an attempt to find an approach which may be generalized to the problem of the scattering from spheres.

The scattering of sound from spheres was first investigated mathematically by Lord Rayleigh (I). Because of the complexity of the solution, he considered only the limiting case where the wavelength of sound was large compared to the radius of the sphere. Morse (2) calculated the solution for rigld immovable spheres, not necessarliy small compared with the wavelength. Faran (3) calculated solutions for elast1c spheres, consldering them neither rigid nor immovable, and, hence, solutions for the inside of the sphere were also obtained. A fen years prior to the publishing of Faran's result, Anderson (4) published results for scattering by a fluid sphere, which represents a slight simplification of the problem since the fluld sphere does not support a shear wave. The solutions he obtained both for the inside and outside of the sphere are complicated in that products and quotients of spherical Bessel and Neumann functions with different arguments occur. Because of this, numerical evaluations were made in computing the scattered acoustic field since little could be done analytically.

Since the exact solutions of Faran and Anderson 
are difficult to deal with both analytically and numerically, 1t 1s useful to attempt to find methods for obtaining approximate results of a more convenient form. In a search for such methods, a problem even simpler than Anderson's may be considered. The purpose of this thesis is to consider the scattering of acoustic pulses and waves from a sphere which has acoustic properties nearly the same as those of the surrounding medium. This allows the expansion of the acoustic parameters inside the sphere by a Taylor series in terms of the acoustic parameters outside the sphere. This approach does permit more to be done analytically to low orders in the expansion than the above mentioned cases.

In Chapter II, the general problem of spherical scattering is considered and the results of Faran and Anderson are obtained for the steady state condition. In Ohapter III, an approximation using the similarity of acoustical properties inside and outside the sphere is made, and the solution is obtained first for arbitrary angles and then for back and forward scattering. The results are compared with those obtalned by the Born approximation. Chapter IV contains the back-scattered solutions for two types of acoust1c pulses. In Chapter $V$, a discussion of the results is given as well as a description of how one would extend the theory. 


\section{CHAPTER II \\ GENERAL SOLUTION FOR SPHERICAI SCATTERING}

It is well known from elastic theory (5) that the displacement $\vec{u}$ can be represented in terms of a scalar potential $\Phi$ and a vector potential $\vec{\psi}$ by the equation

$$
\vec{u}=\nabla \Phi+\nabla \times \vec{\psi}
$$

where $\Phi$ and $\vec{\psi}$ satisfy the following relations

$$
\begin{aligned}
& \frac{1}{c_{L}^{2}} \frac{\partial^{2} \Phi}{\partial t^{2}}=\nabla^{2} \Phi \\
& \frac{1}{c_{\tau}^{2}} \frac{\partial^{2} \vec{\psi}}{\partial t^{2}}=-\nabla \times \nabla \times \vec{\psi}
\end{aligned}
$$

The velocities $C_{L}$ and $C_{T}$ are the velocities of propagation of the longitudinal and transverse waves, respectively, and are defined by the relations

$$
\begin{aligned}
& C_{L}=\sqrt{\frac{\lambda+2 \mu}{\rho}} \\
& C_{T}=\sqrt{\frac{\mu}{\rho}}
\end{aligned}
$$

Consider the solution for a plane wave of engular frequency $\omega$ incident on an elastic sphere. The sphere has a radius $a$, Lamé constants $\lambda_{2}$ and $\mu_{2}$, and 
has a density $\rho_{2}$. Consider the sphere to be immersed In an Infint te ideal fluid with a lame' constant $\lambda_{1}$ $\left(\mu_{1}=0\right.$ for an 1deal fluid) and a density $\rho_{1}$. The Iame constant $\mu$ is the shear modulus and the constant $\lambda$ may be ritten in terms of the bulk modulus $\beta$ and the shear modulus $\mu$ as

$$
\lambda=\beta-\frac{2 \mu}{3}
$$

Let the center of the sphere coincide with the origin of a rectangular coordinate system and let the plane wave approach the sphere along the negative $z$ axis. The spherlcal coordinates used are defined in the usual manner. Due to the cylindrical symmetry about the $Q$ axis, there will be no $\varphi$ dependence in the rcoustic fleld. Also, since no displacements occur in the $\varphi$ direction, the vector potential $\vec{\psi}$ has only a component $\psi_{\varphi}$ in spherical coordinates.

The solutions of Eqns. $(2.2)$ and $(2.3)$ are well known in terms of spherical Bessel functions and Iegendre polynomials. The incoming plane wave is expanded in spherical wave functions by

$$
\Phi_{i}=e^{i(\omega t-k r \cos \theta)}=e^{i \omega t} \sum_{m=0}^{\infty} A_{m}(r) P_{m}(\cos \theta)
$$


6

Upon multiplying by $P_{m}(\cos \theta)$ and then integrating W th respect to $\cos \theta$ from -1 to +1 ,

$$
\int_{-1}^{+1} P_{m}(\cos \theta) e^{-i k r \cos \theta} d(\cos \theta)=A_{m}(r) \int_{-1}^{+1}\left\{P_{m}(\cos \theta)\right\}^{2} d(\cos \theta)
$$

where

$$
\int_{-1}^{+1}\left\{P_{m}(\cos \theta)\right\}^{2} d(\cos \theta)=\frac{2}{2 m+1}
$$

Therefore,

$(2.7)$

$$
A_{m}(r)=\frac{2 m+1}{2} \int_{-1}^{+1} P_{m}(\cos \theta) e^{-i h r \cos \theta} d(\cos \theta)
$$

The scalar potential for the incoming plane wave is then

$$
\Phi_{i}=e^{i \omega t} \sum_{m=0}^{\infty}(-i)^{m}(2 m+1) j m(h, r) P_{m}(\cos \theta)
$$

where

$$
h_{1}=\frac{w}{c_{L_{1}}}
$$


7

Hereafter, for convenience, the time dependence factor $e^{i \omega t}$ wII be understood, but not mitten, in all expressions representing waves.

The scattered outgoing wave is of the form

(2.10)

$$
\Phi_{s}=\sum_{m=0}^{\infty} B_{m} h_{m}^{(2)}(h, r) P_{m}(\cos \theta)
$$

where the $B_{m}$ are constants and $h_{m}^{(2)}(h, r)$ is the spherical Hanker function of second order. The Hanker function of second order appears here to assure that the scattered wave at great distances acts as an outgoing spherical wave, since

$$
h_{m}^{(2)}(k r) \stackrel{r \rightarrow \infty}{\longrightarrow} \frac{e^{-i k r}}{r}
$$

The total scalar potential field outside the sphere is

(2.11)

$$
\Phi^{1}=\sum_{m=0}^{\infty}\left\{(-i)^{m}(2 m+1) j m(h, r)+B_{m} h_{m}^{(2)}(h, r)\right\} P_{m}(\cos \theta)
$$

Since $\mu_{1}=0$ from the definition of an Ideal fluid there is no vector potential outside the sphere.

Inside the sphere, the vector and scalar potentials are

$$
\Phi^{\text {II }}=\sum_{m=0}^{\infty} C_{m} j_{m}\left(k_{2} r\right) P_{m}(\cos \theta)
$$




$$
\psi_{\varphi}^{I I}=\sum_{m=0}^{\infty} D_{m} j_{m}(k, r) \frac{d}{d \theta} P_{m}(\cos \theta)
$$

where

$$
\begin{aligned}
& k_{2}=\frac{\omega}{c_{t_{2}}} \\
& k_{3}=\frac{\omega}{c_{r_{2}}}
\end{aligned}
$$

Spherical Hankel functions do not appear here since they become singular at the origin.

Using the boundary conditions at the surface of the sphere, the coefficlents $B_{m}, C_{m}$ and $D_{m}$ may be determined. These conditions are

1. The normal components of displacement must be continuous.

2. The normal components of stress must be continuous.

3. The tangent1al component of stress must venish.

In spherical coordinates, in terms of $\Phi$ and $\psi_{\phi}$, these three conditions become (3)

$$
u_{r}^{I}=u_{r}^{\text {II }} \text { at } r=a
$$

where

$$
\begin{aligned}
& u_{r}=\frac{\partial \Phi}{\partial r}+\frac{1}{r}\left(\frac{\partial}{\partial \theta}+\frac{\cos \theta}{\sin \theta}\right) \psi_{\varphi} \\
& \sigma_{r r}^{I}=\sigma_{r r}^{\text {II }} \text { at } \quad r=a
\end{aligned}
$$


9

where

$$
\sigma_{r r}=\left(2 \mu \frac{\partial^{2}}{\partial r^{2}}-\frac{\rho \omega^{2} \lambda}{\lambda+2 \mu}\right) \Phi+\frac{2 \mu}{r}\left\{\left(\frac{\partial}{\partial r}-\frac{1}{r}\right) \frac{\partial}{\partial \theta}+\frac{\cos \theta}{\sin \theta}\left(\frac{\partial}{\partial r}-\frac{1}{r}\right)\right\} \psi_{\varphi}
$$

$$
\sigma_{r \theta}^{I I}=0 \text { at } r=a
$$

Where

$$
\begin{aligned}
& \sigma_{r_{\theta}}=\mu\left\{\frac{2}{r}\left(\frac{\partial}{\partial r}-\frac{1}{r}\right) \frac{\partial \Phi}{\partial \theta}+\left(-\frac{\partial^{2}}{\partial r^{2}}+\frac{1}{r^{2}} \frac{\partial}{\partial \theta} \frac{1}{\sin \theta} \frac{\partial}{\partial \theta} \sin \theta\right) \psi_{\varphi}\right\} \\
& \text { respectively. }
\end{aligned}
$$

Upon computing the expressions for the stresses and displacements and substituting them into the boundary requirements, the following equations are obtained which must be satisfied for all $\mathrm{m}$.

$$
(2.14)
$$

$$
\begin{aligned}
& k_{1} B_{m} h_{m}^{\prime}(k, a)-h_{2} C_{m} j_{m}\left(k_{2} a\right)+\frac{m}{a}(m+1) D_{m} j_{m}\left(k_{3} a\right)=-k_{1}(-i)^{m}(2 m+1) j_{m}\left(k_{1} a\right) \\
& \lambda k_{1}^{2} B_{m} h_{m}\left(k_{1} a\right)-h_{2}^{2} C_{m}\left\{\lambda_{2} j m\left(h_{2} a\right)-2 \mu_{2} j m^{\prime \prime}\left(h_{2} a\right)\right\}-\frac{2 \mu_{2} m(m+1)}{a^{2}} D_{m}\left\{k_{3} a j m\left(k_{0} a\right)-j_{m}\left(k_{3} a\right)\right\} \\
& =\lambda \cdot k_{1}^{2}(-i)^{m}(2 m+1) j m(k, a)
\end{aligned}
$$

$$
2 C_{m}\left\{\frac{k_{2}}{a} j m\left(k_{2} a\right)-\frac{1}{a^{2}} j_{m}\left(k_{2} a\right)\right\}+D_{m}\left\{\frac{2}{a^{2}} j_{m}\left(k_{3} a\right)-k_{g^{2}}^{2} j_{m}^{\prime \prime}\left(k_{3} a\right)-\frac{1}{a^{2}} m(m+1) j_{m}\left(k_{3} a\right)\right\}=0
$$

The prime on $h_{m}^{\prime}\left(h_{1} a\right)$ and $j_{m}^{\prime}\left(h_{2} a\right)$ refers to a derivative with respect to the argument and evaluated at $(k, a)$, 
10

$\left(k_{1} a\right)$ or $(k, a)$. The expression for $B_{m}$ is of primary importance now and upon solving the above equations and may be written as

$$
B_{m}=-(-i)^{m}(2 m+1) \text {. }
$$

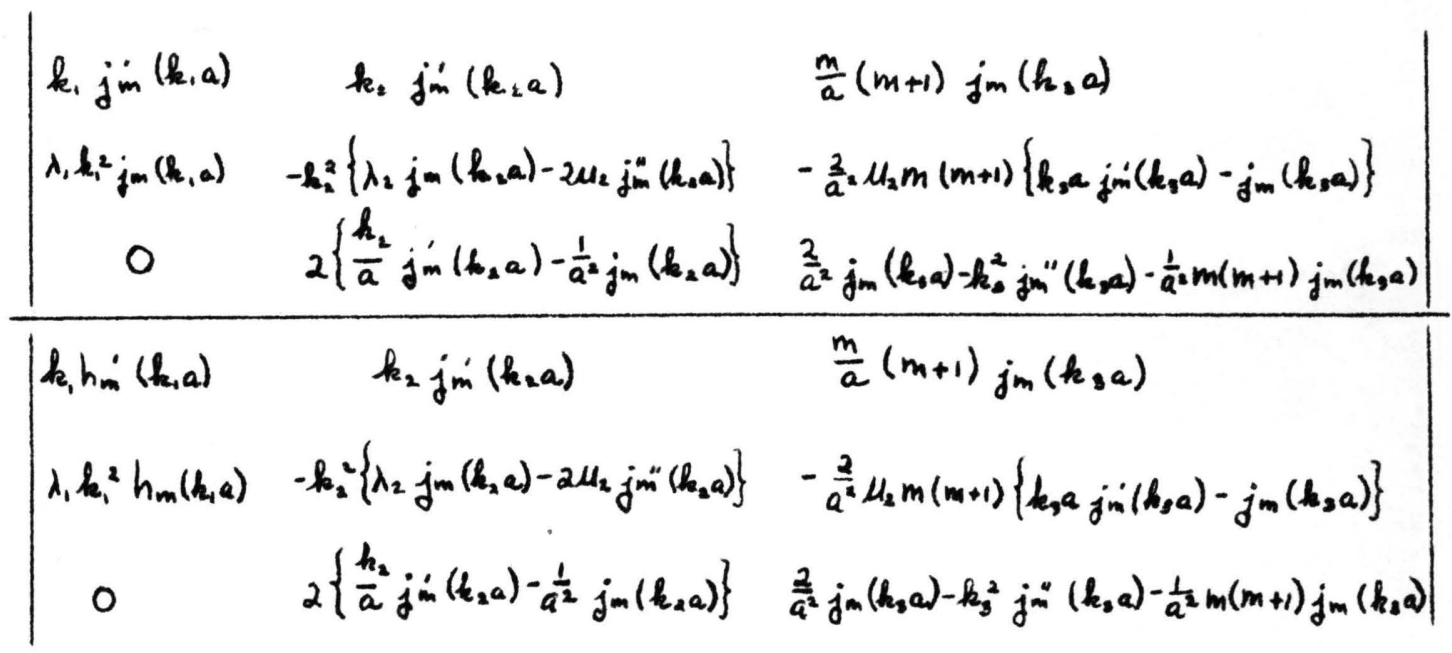

Which is easily shown to be identical with Fran's result (3). The results of Anderson can be obtained from En. (2.15) by taking the limit as $\mu_{2} \rightarrow 0$, or more easily by substituting $\mu=0$ into the boundary conditions. Upon doing the latter, the boundary conditions become

$$
\begin{aligned}
& U_{r}^{I}=U_{r}^{I I} \text { at } r=a \\
& \sigma_{r r}{ }^{I}=\sigma_{r r}^{I} \text { at } r=a
\end{aligned}
$$


where

$$
\begin{aligned}
& u_{r}=\frac{\partial \Phi}{\partial r} \\
& \sigma_{r r}=-\rho \omega^{2} \Phi
\end{aligned}
$$

The third boundary condition in Eqn. (2.13) no longer applies since $\mu_{2}=0$. The two equations to be satisfled nov for a.l $m$ are

$k_{1} B_{m} h_{m}^{\prime}\left(k_{1} a\right)-k_{2} C_{m} j_{m}^{\prime}\left(h_{2} a\right)=-k_{1}(-i)^{m}(2 m+1) j_{m}^{\prime}(k, a)$

$\lambda_{1} h_{1}^{2} B_{m} h_{m}\left(k_{1} a\right)-\lambda_{2} k_{2}^{2} C_{m} j_{m}\left(k_{2} a\right)=-\lambda_{1} k_{1}^{2}(-i)^{m}(2 m+1) j m(k, a)$

Solvine for $B_{m}$,

$$
B_{m}=-(-i)^{m}(2 m+1) \frac{\left|\begin{array}{ll}
k_{1} j_{m}^{\prime}(k, a) & k_{2} j_{m}\left(k_{2} a\right) \\
\lambda_{1} h_{1}{ }^{2} j_{m}(h, a) & \lambda_{2} k_{2}{ }^{2} j m\left(k_{2} a\right)
\end{array}\right|}{\left|\begin{array}{ll}
k_{1} h_{m}^{\prime}(h, a) & k_{2} j_{m}^{\prime}\left(k_{2} a\right) \\
\lambda_{1} k_{1}{ }^{2} h_{m}(k, a) & \lambda_{2} k_{2}{ }^{2} j_{m}\left(k_{2} a\right)
\end{array}\right|}
$$

This expression may easily be shown to be identical with that obtained by Anderson (4). 


\section{CHAPTER III}

AN APPROXIMATION METHOD FOR SCATTERING BY A SPHERE WITH ACOUSTIC PROPERTIES SIMIIAR TO THE SURROUNDING FIUID As calculated in Chapter II, the scattered scalar potential field for a fluid sphere immersed in a fluid medium is

$$
\Phi_{s}=\sum_{m=0}^{\infty} B_{m} h_{m}^{(2)}(k, r) P_{m}(\cos \theta)
$$

where

$$
B_{m}=-(-i)^{m}(2 m+1) \frac{\left|\begin{array}{cc}
k_{1} j_{m}^{\prime}\left(k_{1} a\right) & k_{2} j_{m}^{\prime}\left(k_{1} a\right) \\
\lambda_{1} h_{1}{ }^{2} j m\left(k_{1} a\right) & \lambda_{2} k_{2}{ }^{2} j_{m}\left(k_{2} a\right)
\end{array}\right|}{\left|\begin{array}{ll}
k_{1} h_{m}^{\prime}\left(k_{1} a\right) & k_{2} j_{m}^{\prime}\left(k_{2} a\right) \\
\lambda_{1} k_{1}{ }^{2} h_{m}\left(k_{1} a\right) & \lambda_{2} k_{i}{ }^{2} j_{m}\left(h_{2} a\right)
\end{array}\right|}
$$

For arbitrary $h_{1}$ and $k_{2}$, this expression is difficult to handle analjtically, but numerical calculations may be made for specific values of $k_{1}, k_{2}$ and $a$. If the sphere is acoustically similar to the surrounding medium, $k_{1} \approx k_{2}$ and the functions with the argument $k_{2} a$ may be expanded in a Taylor serles in terms of k,a 
13

by letting

$$
C_{2}=C_{1}(1+\Delta)
$$

where

and

$$
\Delta \ll 1
$$

$$
\rho_{2}=\rho_{1}(1+\delta)
$$

where $\delta \ll 1$.

The function $j_{m}\left(h_{2} a\right)$ may then be expanded to first order in $\Delta$ as

$$
j_{m}\left(k_{2} a\right)=j_{m}\left(k_{1} a\right)-k_{1} a \Delta j_{m}(k, a) .
$$

Similarly, fin ( $\left.h_{2} a\right)$ may be expanded. For convenience, also introduce

$$
\lambda_{2}=\lambda_{1}(1+\epsilon) \text {. }
$$

It is easily shown that

$$
\Delta=\frac{1}{2}(\epsilon-\delta)
$$

Using the above expansions, Equation (2.2) may be written as

$$
B_{m}=-(-i)^{m}(2 m+1) \text {. }
$$

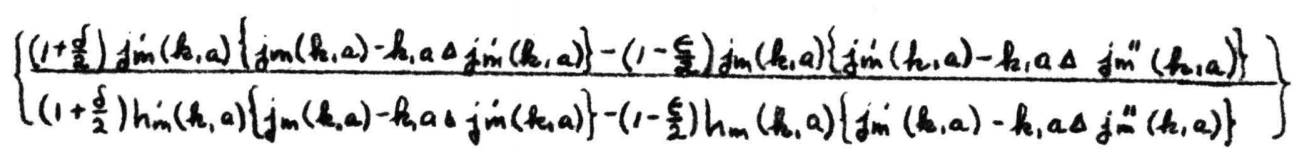


14

Noting that the numerator contains no terms of zero order in $\epsilon$ and $\delta$, the denominator needs only to be taken to zero order in these two quantities.

Rewriting Eq. (3.8) and neglecting all terms with second or higher order in $\epsilon$ and $\delta$ gives

$$
B_{m}=-(-i)^{m}(2 m+1)
$$

$$
\begin{aligned}
& \left\{\frac{\left\{\frac{1}{2}(\epsilon+\delta) j_{m}(k, a) j \dot{m}(k, a)-h, a \Delta j \dot{m}(k, a) j m(h, a)+h, a \Delta j m(k, a) j_{m}^{\prime \prime}(h, a)\right\}}{h_{m}^{\prime}(k, a) j m(k, a)-h_{m}(k, a) j \dot{m}(h, a)}\right\} . \\
& \text { Using the identity } \\
& h_{m}^{\prime}(k, a) j_{m}(h, a)-h_{m}(k, a) j_{m}^{\prime}(k, a)=\frac{-i}{(k, a)^{2}}
\end{aligned}
$$

En. (3.9) becomes

$$
\begin{aligned}
& B_{m}=-i(-i)^{m}(2 m+1)(k a)^{2} . \\
& \left\{\frac{1}{2}(\epsilon+\delta) j m(k, a) j_{m}^{\prime}(h, a)-h_{1} a \Delta j_{m}^{\prime}(h, a) j_{m}^{\prime}(h, a)+h, a \Delta j m(h, a) j_{m}^{\prime \prime}(h, a)\right\}
\end{aligned}
$$

and $\Phi_{s}$ may be written as

$$
\begin{aligned}
\Phi_{s}= & -i(h, a)^{2} \frac{1}{2}(\epsilon+\delta) \sum_{m=0}^{\infty}(-i)^{m}(2 m+1) j_{m}(h, a) j_{m}^{\prime}(k, a) h_{m}^{(2)}(h, r) P_{m}(\cos \theta) \\
& +i(h, a)^{2} \frac{1}{2}(\epsilon-\delta) \sum_{m=0}^{\infty}(-i)^{m}(2 m+1) j_{m}^{\prime}(k, a) j_{m}^{\prime}(h, a) h_{m}^{(2)}(h, r) P_{m}(\cos \theta) \\
& -i(h, a)^{2} \frac{1}{2}(\epsilon-\delta) \sum_{m=0}^{\infty}(-i)^{m}(2 m+1) j_{m}(k, a) j_{m}^{\prime \prime}(h, a) h_{m}^{(2)}(k, r) P_{m}(\cos \theta)
\end{aligned}
$$


15

Using the recursion relation

$$
j_{m}^{\prime}(k a)=\frac{m}{2 m+1} j_{m-1}(k a)-\frac{m+1}{2 m+1} j_{m+1}(k, a)
$$

En. (3.12) becomes

$$
\begin{aligned}
\Phi_{s}= & -i(k a)^{2} \frac{1}{2}(\epsilon+\delta) \sum_{m=0}^{\infty}(-i)^{m} m j_{m}\left(h_{a}\right) j_{m-1}(k a) h_{m}^{(2)}(k r) P_{m}(\cos \theta) \\
& +i(k a)^{2} \frac{1}{2}(\epsilon+\delta) \sum_{m=0}^{\infty}(-i)^{m}(m+1) j_{m}(k, a) j_{m+1}(k a) h_{m}^{(2)}(k r) P_{m}(\cos \theta) \\
& +i(k a)^{3} \frac{1}{2}(\epsilon-\delta) \sum_{m=0}^{\infty}(-i)^{m} \frac{m^{2}}{2 m+1} j_{m-1}^{2}(k a) h_{m}^{(2)}(k r) P_{m}(\cos \theta) \\
& -i(k a)^{3} \frac{1}{2}(\epsilon-\delta) \sum_{m=0}^{\infty}(-i)^{m} \frac{2 m(m+1)}{2 m+1} j_{m+1}(k a) j_{m-1}(k a) h_{m}^{(2)}(k r) P_{m}(\cos \theta) \\
& +i(k a)^{3} \frac{1}{2}(\epsilon-\delta) \sum_{m=0}^{\infty}(-i)^{m} \frac{(m+1)^{2}}{2 m+1} j_{m+1}^{2}(k a) h_{m}^{(2)}(k r) P_{m}(\cos \theta) \\
& -i(k a)^{3} \frac{1}{2}(\epsilon-\delta) \sum_{m=0}^{\infty}(-i)^{m} \frac{m(m-1)}{2 m-1} j_{m}(k a) j_{m-2}(k a) h_{m}^{(2)}(k r) P_{m}(\cos \theta) \\
& +i(k a)^{3} \frac{1}{2}(\epsilon-\delta) \sum_{m=0}^{\infty}(-i)^{m} \frac{m^{2}}{2 m-1} j_{m}^{2}\left(k_{a} a\right) h_{m}^{(2)}(k r) P_{m}(\cos \theta) \\
& +i(k a)^{3} \frac{1}{2} \frac{1}{2}(\epsilon-\delta) \sum_{m=0}^{\infty}(-i)^{m} \frac{(m+1)(m+2)}{2 m+3} j_{m=0}^{\infty}(-i)^{m} \frac{(m+1)^{2}}{2 m+3} j_{m}^{2}(h a) h_{m}^{(2)}\left(k_{r} r\right) P_{m}(\cos \theta) \\
& +(k a) h_{m}^{(2)}(k r) P_{m}(\cos \theta)
\end{aligned}
$$


16

This represents the scattered scalar potential field for arbitrary angles.

Perhaps the most useful and interesting result is for $\theta=\pi$, ide., back-scattering which will now be considered.

For convenience the far field will be examined which allows the Hanker function $h_{m}^{(2)}(k r)$ to be replaced by its asymptotic form for large $r$

$$
h_{m}^{(2)} \stackrel{r \rightarrow \infty}{\longrightarrow} \frac{e^{-i k r}}{k r} i^{m+1}
$$

For back-scattering,

$$
P_{m}(\cos \theta)=(-1)^{m}
$$

Therefore, Equation (3.14) becomes

$$
\begin{aligned}
\Phi_{s} & =\frac{e^{-i k r}}{k r}(k a)^{2} \frac{1}{2}(\epsilon+\delta) \sum_{m=0}^{\infty} m j_{m}(k a) j_{m-1}(k a)(-1)^{m} \\
& -\frac{e^{-i k r}}{k r}(k a)^{2} \frac{1}{2}(\epsilon+\delta) \sum_{m=0}^{\infty}(m+1) j_{m}(k a) j_{m+1}(k a)(-1)^{m} \\
& -\frac{e^{-i k r}}{k r}(k a)^{3} \frac{1}{2}(\epsilon-\delta) \sum_{m=0}^{\infty} \frac{m^{2}}{2 m+1} j_{m-1}^{2}(k a)(-1)^{m} \\
& +\frac{e^{-i k r}}{k r}(k a)^{3} \frac{1}{2}(\epsilon-\delta) \sum_{m=0}^{\infty} \frac{2 m(m+1)}{2 m+1} j_{m-1}(k a) j_{m+1}(k a)(-1)^{m} \\
& -\frac{e^{-i k r}}{k r}(k a)^{3} \frac{1}{2}(\epsilon-\delta) \sum_{m=0}^{\infty} \frac{(m+1)^{2}}{2 m+1} j_{m+1}^{2}(k a)(-1)^{m}
\end{aligned}
$$




$$
\begin{aligned}
& +\frac{e^{-i k r}}{k r}(k a)^{3} \frac{1}{2}(\epsilon-\delta) \sum_{m=0}^{\infty} \frac{m(m-1)}{2 m-1} j_{m}(k a) j_{m-2}(k a)(-1)^{m} \\
& -\frac{e^{-i k r}}{k r}(k a)^{3} \frac{1}{2}(\epsilon-\delta) \sum_{m=0}^{\infty} \frac{m^{2}}{2 m-1} j_{m}{ }^{2}(k a)(-1)^{m} \\
& -\frac{e^{-i k r}}{k r}(k a)^{3} \frac{1}{2}(\epsilon-\delta) \sum_{m=0}^{\infty} \frac{(m+1)^{2}}{2 m+3} j_{m}^{2}(k a)(-1)^{m} \\
& +\frac{e^{-i k r}}{k r}(k a)^{3} \frac{1}{2}(\epsilon-\delta) \sum_{m=0}^{\infty} \frac{(m+1)(m+2)}{2 m+3} j_{m}(k a) j_{m+2}(k a)(-1)^{m}
\end{aligned}
$$

It is seen immediately that when the fifth and seventh, third and elghth and, the fourth, slxth and ninth terms respectively are added together their sums are zero, leaving

$\Phi_{s}=\frac{e^{-i k r}}{k r}(k a)^{2}(\epsilon+\delta) \sum_{m=0}^{\infty} m_{j m}\left(k_{a}\right) j_{m-1}(k a)(-1)^{m}$

The evaluation of this infinite sum is found in Append1x (A) and gives

$$
\Phi_{s}=-\frac{e^{-i k r}}{k r}(k a)^{2} \frac{1}{2}(\epsilon+\delta) j_{1}(2 k a)
$$

Using the asymptotic expression for $2 k a \ll 1$ Eqn. (3.17) reduces to

$$
\Phi_{s}=-\frac{h^{2} a^{3}}{3 r}(\epsilon+\delta)
$$


wich agrees with the Rayleigh limit for small spheres (1).

For forward scattering, it may be shown that

$$
\Phi_{s}=-\frac{e^{-i k r}}{k r}(k a)^{3}(\epsilon-\delta) \sum_{m=0}^{\infty}(2 m+1) j_{m}^{\prime 2}(k a)
$$

This expression also agrees with the Rayleigh limit for forward scattering. slnoe only two terms of the Taylor expansion of $j_{m}\left(h_{2} a\right)$ are used, some amount of error is involved in the calculations, but since the spherlcal Bessel functions are vell behaved, it is expected that the errors are small if the term $k, a \Delta$ is small. To determine the magnitude of these errors it is convenient to calculate a few numerical values. The expansion requires the term $h, a \Delta$ to be very much less than one, so a value of 0.1 was used for convenience and typloal values of $\mathrm{ka}$ were chosen. Frrors of the order of one percent of the quantity k,as were found to exist but this quantity has already been assumed to be very small. Therefore, to a sultable degree of approximation, the errors may be assuned negligible by choosing k,as appropriately small.

When the scattering can be considered weak the Born approximation (7) is another approach which may be used to compute the scattering of an acoustical wave. 
The results of this approach, as calculated in Appendix

(c) Gives the scattered field

$$
\Phi_{s}=\frac{e^{-i k r}}{8 k r}(\delta-\epsilon)\{\operatorname{Sin} 2 k a-2 k a \cos 2 k a\} \text {. }
$$

The result of the approximation method used in this chapter gives

$$
\Phi_{s}=-\frac{e^{-i k r}}{2 r k}(k a)^{2}(\epsilon+\delta) j_{j}(2 k a)
$$

which may be written as

$$
\Phi_{s}=-\frac{e^{-i k r}}{8 k r}(\epsilon+\delta)\{\sin 2 k a-2 k a \cos 2 k a\} \text {. }
$$

Comparing the two solutions, it is seen that they are ident1cal with the exception of the sign of $\delta$. In the Rayleigh Ilnit, \$gn. (3.22) agrees with Rayleigh's result. However, taking the Raylelgh limit of the Born approximation still leaves the slen of $\delta$ different from Rayleigh's result. One possible explanation of the difference in the sign of $\delta$ is that the Born approximation tacitly assumes that the scattering region does not move during the time interval the wave is being scattered. However, an incoming oscillating wave will cause the sphere to vibrate about its rest position in a complicated vibrational pattern. This motion gives rise 
20

to a sound field which is Included in the exact calculation but not in the Born approximation. Since the difference in $\mathrm{s} 1 \mathrm{gn}$ is associated w th the inertial rather than the compressibility term, this explanation seems at least plausible. A detailed study of this difference of results is now being carried out and will be reported in a later publication. 


\section{CHAPTER IV}

\section{THE BACK-SCATTERING OF PUISES FROM FIUID SPHERES}

The expansion of the acoustical properties Inside the sphere in terns of the acoustical properties outside the sphere allows the steady state solution of the wave equation to be mitten in a very simple form. Having the back-scattered steady state solution in this form permits the back-scattered flelds for acoustic pulses to be calculated quite easily. The types of pulses considered here are an exponential pulse and a sinusoldally varying pulse of short auration. The interest in these two types of pulses arisea from the fact that they are the most frequently used in acoustic work.

The back-scattered field for an arbitrary incoming pulse $s(t)$ is

$$
\Phi=-\frac{a^{2}}{2 \sqrt{2 i} c r}(\epsilon+\delta) \int_{-\infty}^{+\infty} e^{i \omega t} \omega g(\omega) j_{1}(2 k a) d \omega
$$

Where $g(w)$ is the Fourler transform defined in Append1x B。

\section{Exponential Pulse}

The Fourier transform for this type of pulse is calculated in Appendix $B$ and is

$$
g(w)=\frac{-i}{\sqrt{2 \pi}(w-i \sigma)}
$$


22

Using the identity

$$
j^{\prime}(2 k a)=\frac{\sin 2 k a}{(2 k a)^{2}}-\frac{\cos 2 k a}{2 k a}
$$

and then expanding the trigonometric functions in terms of exponentials, $\Phi$ becomes the sum of four integrals

$$
\phi=I_{1}+I_{2}+I_{3}+I_{4}
$$

Where

$$
\begin{aligned}
& I_{1}=-\frac{c}{32 \pi r}(\epsilon+\delta) \int_{-\infty}^{+\infty} \frac{e^{i \omega\left(t^{\prime}+\frac{2 a}{c}\right)} d \omega}{\omega(\omega-i \sigma)} \\
& I_{2}=\frac{c}{32 \pi r}(\epsilon+\delta) \int \frac{e^{i \omega\left(t^{\prime}-\frac{2 a}{c}\right)}}{\omega(\omega-i \sigma)} d \omega \\
& I_{3}=\frac{a i}{16 \pi r}(\epsilon+\delta) \int_{-\infty}^{+\infty} \frac{e^{i \omega\left(t^{\prime}+\frac{2 a}{\varepsilon^{2}}\right)} d \omega}{\omega-i \sigma} d \omega \\
& I_{4}=\frac{a i}{16 \pi r}(\epsilon+\delta) \int \frac{e^{i \omega\left(t^{\prime}-\frac{2 a}{c}\right)} d \omega}{\omega-i \sigma}
\end{aligned}
$$

and where

$$
t^{\prime}=t-\frac{r}{c}
$$

Evaluating these integrals by contour

integration, the following results are obtained for the 
23

time intervals indicated:

$$
I_{1}=-\frac{c}{16 r \sigma}(\epsilon+\delta)\left\{e^{-\sigma\left(t^{\prime}+\frac{2 a}{c}\right)}-\frac{1}{2}\right\}
$$

$$
I_{1}=-\frac{c}{32 r \sigma}(\epsilon+\delta)
$$

$$
I_{2}=\frac{c}{16 r \sigma}(\epsilon+\delta)\left\{e^{-\sigma\left(t^{\prime}-\frac{2 a}{c}\right)}-\frac{1}{2}\right\}
$$

$$
I_{2}=\frac{c}{32 r \sigma}(\epsilon+\delta)
$$

$$
I_{3}=-\frac{a}{8 r}(\epsilon+\delta) e^{-\sigma\left(t^{\prime}+\frac{2 a}{c}\right)}
$$

$$
I_{3}=0
$$

$$
I_{4}=-\frac{a}{8 r}(\epsilon+\delta) e^{-\sigma\left(t^{\prime}-\frac{2 a}{c}\right)}
$$

$$
I_{4}=0
$$

$$
t^{\prime}+\frac{2 a}{c}>0
$$$$
t^{\prime}+\frac{2 a}{c}<0
$$

$$
t^{\prime}-\frac{2 a}{c}>0
$$

$$
t^{\prime}-\frac{2 a}{c}<0
$$

$$
t^{\prime}+\frac{2 a}{c}>0
$$

$$
t^{\prime}+\frac{2 a}{c}<0
$$

$$
\begin{aligned}
& t^{\prime}-\frac{2 a}{c}>0 \\
& t^{\prime}-\frac{2 a}{c}<0
\end{aligned}
$$

To clearly see the total scattered field for the different time intervals, it is convenient to construct the following table. 


$$
\phi=
$$

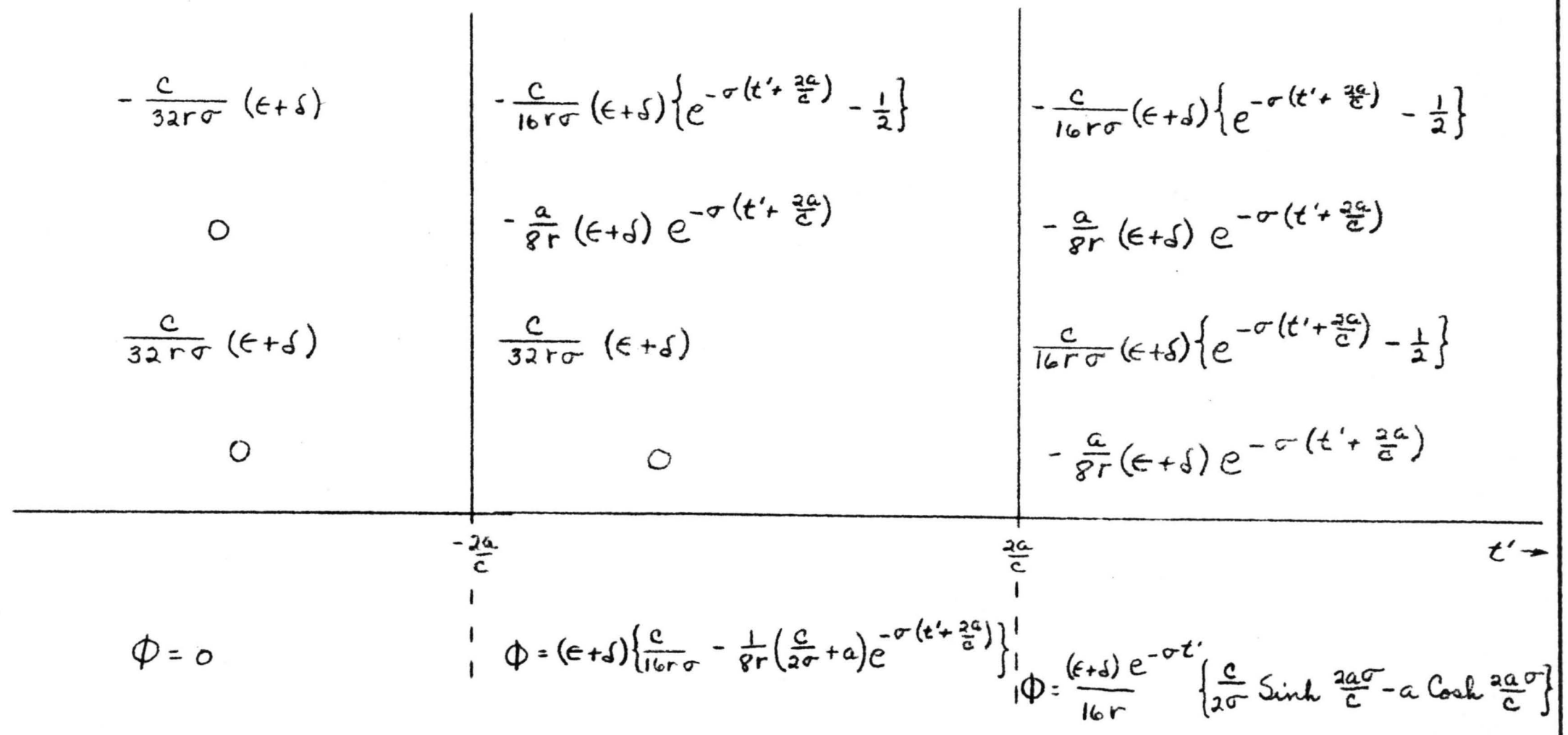

$\stackrel{\sim}{+}$ 
When the values of the integrals are summed in the respective time intervals the following results are obtained:

$$
\begin{aligned}
& \text { (a) } t^{\prime}<-\frac{2 a}{c} \text {. The total back-scattered } \\
& \text { field for this time interval } \\
& \text { is zero as would be expected. } \\
& \text { (b) }-\frac{2 a}{c}<t^{\prime}<\frac{2 a}{c} \text { The total back-scattered } \\
& \text { fleld for this time interval } \\
& \text { 1s: } \\
& \Phi=(\epsilon+\delta)\left\{\frac{c}{16 r \sigma}-\frac{1}{8 r}\left(\frac{c}{2 \sigma}+a\right) e^{-\sigma\left(t^{\prime}+\frac{2 a}{c}\right)}\right\}
\end{aligned}
$$

\section{1, :}

$$
\phi=e^{-\sigma t^{\prime}}\left(\frac{d+\epsilon}{16 r}\right)\left\{\frac{c}{2 \sigma} \operatorname{Sinh} \frac{2 a \sigma}{c}-a \cosh \frac{2 a \sigma}{c}\right\}
$$

No time independent term occurs here and the time dependent term appears to have been scattered from the back surface of the sphere. 
26

For the sinusoidal pulse, the acoustical disturbance is

$$
S(t)=\sin \omega \frac{t}{\tau}=\sin \omega_{0} t \quad-\frac{T}{2}<t<\frac{T}{2}
$$

and

$$
S(t)=0 \quad t>\frac{T}{2}, t<-\frac{T}{2} .
$$

The Fourier transform for this pulse as calculated in Appendix B is

$$
g(\omega)=\frac{-2 i \omega_{0} \sin \omega \frac{T}{2}}{\sqrt{2 \pi}\left(\omega-\omega_{0}\right)\left(\omega+\omega_{0}\right)}
$$

Expanding $\sin \omega \frac{T}{2}$ in exponential and substituting En. (4.8) into En. (4.1) the backscattered field $\Phi$ becomes the sum of eight integrals

$$
\phi=I_{1}+I_{2}+\cdots+I_{8}
$$

where

$$
\begin{aligned}
& I_{1}=-\gamma \int_{-\infty}^{+\infty} \frac{e^{i \omega\left(t^{\prime}+\frac{2 a}{c}+\frac{T}{2}\right)} d \omega}{\omega\left(\omega-\omega_{0}\right)\left(\omega+\omega_{0}\right)} \\
& I_{2}=\gamma \int_{-\infty}^{+\infty} \frac{e^{i \omega\left(t^{\prime}+\frac{2 a}{c}-\frac{T}{2}\right)} d \omega}{\omega\left(\omega-\omega_{0}\right)\left(\omega+\omega_{0}\right)}
\end{aligned}
$$


27

$$
\begin{aligned}
& I_{3}=f \int_{-\infty}^{+\infty} \frac{e^{i \omega\left(t^{\prime}-\frac{2 a}{c}+\frac{T}{2}\right)} d \omega}{\omega\left(\omega-\omega_{0}\right)\left(\omega+\omega_{0}\right)} \\
& I_{4}=-j \int_{-\infty}^{+\infty} \frac{e^{i \omega\left(t^{\prime}-\frac{2 a}{c}-\frac{T}{2}\right)} d \omega}{\omega\left(\omega-\omega_{0}\right)\left(\omega+\omega_{0}\right)} d \omega \\
& I_{5}=\frac{2 a i}{c} j \int_{-\infty}^{+\infty} \frac{e^{i \omega\left(t^{\prime}+\frac{2 a}{c}+\frac{T}{2}\right)} d \omega}{\left(\omega-\omega_{0}\right)\left(\omega+\omega_{0}\right)} d \omega \\
& I_{6}=-\frac{2 a i}{c} j \int_{-\infty}^{+\infty} \frac{e^{i \omega\left(t^{\prime}+\frac{2 a}{c}-\frac{T}{2}\right)}}{\left(\omega-\omega_{0}\right)\left(\omega+\omega_{0}\right)} d \omega \\
& I_{7}=\frac{2 a i}{c} j \int_{-\infty}^{+\infty} \frac{e^{i \omega\left(t^{\prime}-\frac{2 a}{c}+\frac{T}{2}\right)} d \omega}{\left(\omega-\omega_{0}\right)\left(\omega+\omega_{0}\right)} \\
& I_{8}=-\frac{2 a i}{c} j \int_{-\infty}^{+\infty} \frac{e^{i \omega\left(t^{\prime}-\frac{2 a}{c}-\frac{T}{2}\right)}}{\left(\omega-\omega_{0}\right)\left(\omega+\omega_{0}\right)} d \omega
\end{aligned}
$$

where

$$
J=\frac{c \omega_{0}}{32 \pi i r}(\epsilon+\delta)
$$

Evaluating the integrals by the contour

integration given in Append ix B, the integrals have the following values for the time intervals indicated: 
28

$$
\begin{array}{ll}
I_{1}=\frac{(\epsilon+\delta) c}{32 \omega_{0} r}\left\{1-\cos \omega_{0}\left(t^{\prime}+\frac{2 a}{c}+\frac{T}{2}\right)\right\} & t^{\prime}+\frac{2 a}{c}+\frac{T}{2}>0 \\
I_{1}=\frac{(\epsilon+\delta) c}{32 \omega_{0} r}\left\{\cos \omega_{0}\left(t^{\prime}+\frac{2 a}{c}+\frac{T}{2}\right)-1\right\} & t^{\prime}+\frac{2 a}{c}+\frac{T}{2}<0 \\
I_{2}=\frac{(\epsilon+\delta) c}{32 \omega_{0} r}\left\{\cos \omega_{0}\left(t^{\prime}+\frac{2 a}{c}-\frac{T}{2}\right)-1\right\} & t^{\prime}+\frac{2 a}{c}-\frac{T}{2}>0 \\
I_{2}=\frac{(\epsilon+\delta) c}{32 \omega_{0} r}\left\{1-\cos \omega_{0}\left(t^{\prime}+\frac{2 a}{c}-\frac{T}{2}\right)\right\} & t^{\prime}+\frac{2 a}{c}-\frac{T}{2}<0 \\
I_{3}=\frac{(\epsilon+\delta) c}{32 \omega_{0} r}\left\{\cos \omega_{0}\left(t^{\prime}-\frac{2 a}{c}+\frac{T}{2}\right)-1\right\} & t^{\prime}-\frac{2 a}{c}+\frac{T}{2}>0 \\
I_{5}=-\frac{(\epsilon+\delta) c}{16 r} \sin \omega_{0}\left(t^{\prime}+\frac{2 a}{c}+\frac{T}{2}\right) & t^{\prime}+\frac{2 a}{c}+\frac{T}{2}>0 \\
I_{4}=\frac{(\epsilon+\delta) c}{32 \omega_{r}}\left\{\cos \omega_{0}\left(t^{\prime}-\frac{2 a}{c}-\frac{T}{2}\right)-1\right\} & t^{\prime}-\frac{2 a}{c}-\frac{T}{2}<0 \\
I_{4}=\frac{(\epsilon+\delta) c}{32 \omega_{0} r}\left\{1-\cos \omega_{0}\left(t^{\prime}-\frac{2 a}{c}-\frac{T}{2}\right)\right\} & t^{\prime}-\frac{2 a}{c}+\frac{T}{2}<0
\end{array}
$$


29

$I_{5}=\frac{(\epsilon+\delta) a}{16 r} \operatorname{Sin} \omega_{0}\left(t^{\prime}+\frac{2 a}{c}+\frac{I}{2}\right)$

$I_{6}=\frac{(\epsilon+\delta) a}{16 r} \sin \omega_{0}\left(t^{\prime}+\frac{2 a}{c}-\frac{T}{2}\right)$

$I_{6}=\frac{-(\epsilon+\delta) a}{16 r} \operatorname{Sin} \omega_{0}\left(t^{\prime}+\frac{2 a}{c}-\frac{T}{2}\right)$

$I_{1}=\frac{-(t+\delta) a}{16 r} \sin \omega_{0}\left(t^{\prime}-\frac{2 a}{c}+\frac{T}{2}\right)$

$I_{1}=\frac{(\epsilon+\delta) a}{16 r} \operatorname{Sin} \omega_{0}\left(t^{\prime}-\frac{2 a}{c}+\frac{T}{2}\right)$

$I_{8}=\frac{(\epsilon+\delta) a}{16 r} \operatorname{Sin} \omega_{0}\left(t^{\prime}-\frac{2 a}{c}-\frac{T}{2}\right)$

$I_{8}=\frac{-(\epsilon+\delta) a}{16 r} \operatorname{Sin} \omega_{0}\left(t^{\prime}-\frac{2 a}{c}-\frac{T}{2}\right)$ $t^{\prime}+\frac{2 a}{c}+\frac{T}{2}<0$

$t^{\prime}+\frac{2 a}{c}-\frac{T}{2}>0$

$t^{\prime}+\frac{2 a}{c}-\frac{T}{2}<0$

$t^{\prime}-\frac{2 a}{c}+\frac{T}{2}>0$

$t^{\prime}-\frac{2 a}{c}+\frac{T}{2}<0$

$t^{\prime}-\frac{2 a}{c}-\frac{T}{2}>0$

$t^{\prime}-\frac{2 a}{c}-\frac{T}{2}<0$

The results of this type of pulse are tabulated in the following table. 


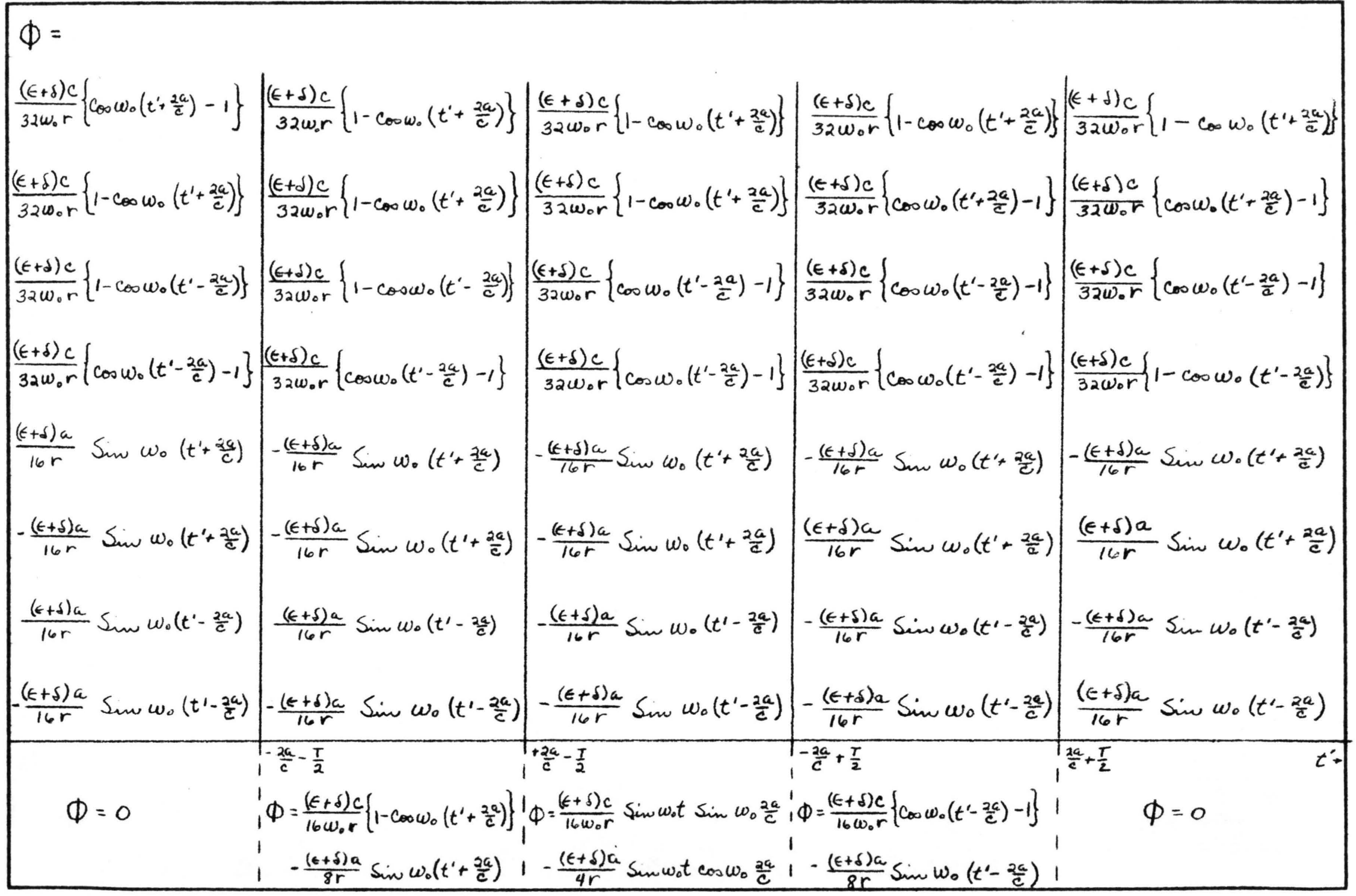


When the values of the integrals are summed for each time interval, the following results are obtained:

(a) $t^{\prime}<-\frac{2 a}{c}-\frac{T}{2}$, As expected, the total back-scattered field for

this time interval is zero.

(b)

The total back-scattered $-\frac{2 a}{c}-\frac{T}{2}<t^{\circ}<\frac{2 a}{c}-\frac{T}{2}$, field in this time intervaf 15:

$\Phi=(\epsilon+\delta)\left\{\frac{c}{16 \omega_{0} r}\left(1-\cos \omega_{0}\left(t^{\prime}+\frac{2 a}{c}\right)\right)-\frac{a}{8 r} \sin \omega_{0}\left(t^{\prime}+\frac{2 a}{c}\right)\right\}$

This field appears to be the first of the wave train being scattered by the front surface of the sphere, and, as in the exponential pulse case, a time independent term appears.

(c) $2 a-T<t^{\prime}, \frac{T}{2} 2 a$ The total back-scattered $\frac{2 a}{c}-\frac{T}{2}<t^{\prime}<\frac{T}{2}-\frac{2 a}{c}$, field in this time interval 1s:

$\Phi=(\epsilon+\delta) \frac{\sin \omega_{0} t}{4 r}\left\{\frac{c}{4 \omega_{0}} \sin \omega_{0} \frac{2 a}{c}-a \cos \omega_{0} \frac{2 a}{c}\right\}$

This fleld appears to be the first of the pulse being scattered from the back surface of the sphere. 
(d)

$$
\begin{aligned}
& -\frac{2 a}{c}+\frac{T}{2}<t<\frac{T}{2}+\frac{2 a}{c}, \\
& \text { 1s: } \\
& \phi=\frac{(\epsilon+\delta) c}{16 \omega_{0} r}\left\{\cos \omega_{0}\left(t^{\prime}-\frac{2 a}{c}\right)-1\right\}-\frac{(\epsilon+\delta) a}{8 r} \sin \omega_{0}\left(t^{\prime}-\frac{2 a}{c}\right)
\end{aligned}
$$

(e)

The total back-scattered $t^{\prime}>\frac{T}{2}+\frac{2 a}{C}$ field in this time intervaf is again zero as would be expected. Some care must be taken in ohoosing a pulse since the method of analysis used in this thesis has some Iimitations. First, since the asymptotic limit of the spherical Hankel function becomes singular at $w=0$, the pulse must be chosen such that its Fourler tronsform has a small contribution of frequencles near $\omega=0$. It may also be shown that the integrand vanishes at $\omega=0$ even when $h_{m}^{(2)}(h, r)$ is not used in 1ts asymptotic form; thus the error due to representing $h^{(2)}\left(k_{r}\right)$ as $\frac{e^{-i k r}}{k r}$ is not large. Secondly, since the Taylor expansion requires $k, a \Delta \ll 1$, the Fourler transform of the pulse must not contain frequencies such that the inequallty cannot be satisfied with an adjustment of $a, \Delta$ or $c$. Also, 
since $k, a \Delta \ll 1$ and since frequencies near $\omega=0$ are not alloved, pulses must be chosen such that with an adjustment of $a, \Delta$ and $c$, the inequality is satisfied without much contribution from frequencies near $\omega=0$. 


\section{CHAPTER $V$ \\ SUMMARY AND DISOUSSION}

In this thesis it has been shown that the scattering of sound by fluld spheres immersed in an infinite fluid may be calculated easily by assuming that the acoustical properties of the sphere are very similer to the acoustical properties of the surrounding fluid. This allows the expansion of functions involving the acoustical parameters inside the sphere in terms of a Taylor serles in which the acoustical parameters outslde the sphere appear. This, in turn, allows the backscattered field for arbitrary pulses to be easily celculated at least to first order in the expansion. The first order back-scattered fleld for the two types of pulses considered appears, from time of arrival considerations, to consist of a pulse being scattered from the front surface and one from the back surface of the sphere. For higher accuracy more terms would need to be used in the expansion. In order to see the result of including higher order terms the contribution due to second order terms may easily be determined. The second order terms in $\epsilon$ and $\delta$ that appear in the scattering coefficient $B_{m}$ are 


$$
\begin{aligned}
& -\frac{\delta(\epsilon+\delta)}{4} j_{m}^{2}(k a) j m(k a) h_{m}(k a)-\frac{\epsilon(\epsilon+\delta)}{4} j_{m}(k a) j_{m}^{2}(k a) h_{m}(h a) \\
& -\frac{k a}{4}(\epsilon-\delta)(\epsilon+\delta) j_{m}\left(k_{a}\right) j_{m}^{\prime}(k a)\left\{j_{m}^{\prime \prime}(k a) h_{m}(k a)-j_{m}^{\prime}(k a) h_{m}^{\prime}(k a)\right\} \\
& -\frac{i(\epsilon+\delta)^{2}}{8}\left(j m_{j \prime \prime}^{\prime \prime}(k a) j m(k a)-j m(k a) j_{m}^{\prime \prime}(k a)\right)+\frac{i}{4 k a}(\epsilon+\delta)\left(\delta j_{j m}^{\prime 2}(k, a)+\epsilon j m(k a) j_{m}^{\prime \prime}(k, a)\right) \\
& -\frac{k a}{4} \delta(\epsilon-\delta) j_{m}(k a) h_{m}^{\prime}(k a)\left\{j_{m}(k a) j m^{\prime \prime}(k a)-j m^{\prime 2}(k a)\right\} \\
& -\frac{k g}{4} \epsilon(\epsilon-\delta) h_{m}(k a) j_{m}^{\prime}(k a)\left\{j m(k a) j m(k a)-j m^{2}(k a)\right\}
\end{aligned}
$$

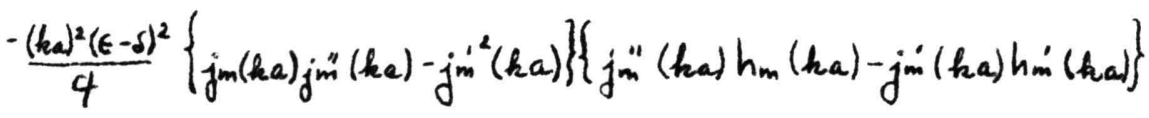

All these terms contain products of either two or four spherical Bessel functions. When the time dependences are investigated by means of contour integration similar to that appearing in Append1x B, it appears that some of the terms represent the pulse being scattered by the front and back surfaces of the sphere just as in the first order case. However, the other terms possess time dependences wich seem to have no simple geometric interpretation. The orlgin of all these reflected 
pulses is not adequately knom and it is very doubtful that any of them have such simple geometric origins then one considers the ratio of wavelength to sphere s1ze. Further investigation of the dynamic behavior of the sphere itself would be of interest. Due to the complexity of this problem, time has not allowed for its inclusion in this thesis.

The existence of time independent terms for the back-scattered fleld produced by the two pulses is not completely understood at this time and subsequent investigation related to that mentioned above may provide information on the origin of the terms. Since the time between the arrivals of the two scattered pulses discussed in Chapter IV is relatively short, one possible explanation for the terms whlch appear to be time independent is that they may represent the first term in the expansion of a slowly varying time dependent function. The assumption that the acoustical properties of the sphere are very similar to the acoustical properties of the surrounding fluid also allows the Born approximation to be used to compute the scattering of a plane wave. However, when the results of the exact calculation and the Born approximation are compared, it is seen that there exists a disagreement in the sign of $\delta$ for back-scattering and an even more complicated discrepancy for other angles of scattering. In Chapter 
III, It was suggested that this disagreement may be attributed to the fact that the Born approximation tacitly assumes that the scattering region does not move during the time interval the wave is being scattered. The exact solution, however, does take into account the movement of the sphere. Further investigation of this problem 1s under way.

The problem consldered here has led to an easier way to obtaln the approximate fleld due to scattering by spheres at least in the limit of small differences in the elast1c constants as described above. It is hoped that the extension of this method to higher order terms will be of general use in scattering problems throughout physics. 
APPENDIX A

\section{EVAIUUATON OF INEINITE SUM}

The infinite sum to be evaluated is

$$
T=\sum_{m=0}^{\infty}(-1)^{m} m j_{m}(h \omega)_{j m-1}(h a)
$$

Expressing the spherlcel Bessel functions as cylindrical Bessel functions by

$$
j_{m}(k a)=\sqrt{\frac{\pi}{2 k a}} J_{m+1 / 2}(k a)
$$

and using the 1dentity (8)

$$
J_{\mu}(k a) J_{\nu}(k a)=\frac{2}{\pi} \int_{0}^{\pi / 2} J_{\mu+\nu}(2 k \omega \cos \theta) \cos (u-\nu) \theta d \theta
$$

Equation $(\mathrm{A}-1)$ becomes

$$
T=\frac{1}{k a} \sum_{m=0}^{\infty}(-1)^{m} m \int_{0}^{\pi / 2} J_{2 m}(2 k a \cos \theta) \cos \theta d \theta
$$

Since $J_{2 m}(2 k a \cos \theta)$ is a well behaved function, the Interchange of the summation and integration operations presents no difficulty. Also, the first term of the summation vanishes so the summation may be started from one instead of zero. 
39

Rewriting Equation (A-4)

$$
T=\frac{1}{k a} \int_{0}^{\pi / 2} \cos \theta \sum_{m=0}^{\infty}(-1)^{m} m J_{2 m}(2 k a \cos \theta) d \theta
$$

$(A-5)$

A recursion relationship of use at this point is

(8)

$$
J_{m}(z)=\frac{z}{2 m} J_{m-1}(z)+\frac{z}{2 m} J_{m+1}(z)
$$

and when used in Equation (A-5) gives

$(A-7)$

$$
T=\frac{1}{2} \int_{0}^{\pi / 2} \cos ^{2} \theta\left\{\sum_{m=0}^{\infty}(-1)^{m} J_{2 m-1}(2 k a \cos \theta)+\sum_{m=1}^{\infty}(-1)^{m} J_{2 m+1}(2 k a \cos \theta)\right\} d \theta
$$

Examining the terms in the bracket it is seen that the sum of the infinite series is equal to

$$
\sum_{m=1}^{\infty} J_{2 m-1}(2 k a \cos \theta)+\sum_{m=1}^{\infty}(-1)^{m} J_{2 m+1}(2 k a \cos \theta)=-J_{1}(2 k a \cos \theta)
$$

Therefore,

$$
T=-\frac{1}{2} \int_{0}^{\hat{y} / 2} J_{1}(2 k a \cos \theta) \cos ^{2} \theta d \theta
$$

$(A-8)$

Another Identity of use here is (8)

$$
J_{\nu+\mu+1}(2 z)=\frac{(2 z)^{\nu+1}}{2^{\nu} \Gamma(\nu+1)} \int_{0}^{\pi / 2} J_{\mu}(2 z \sin \theta \theta) \sin ^{\mu+1} \theta \cos ^{2 \nu+1} \theta d \theta
$$

$(A-9)$ 
40

By letting $\theta=\Phi+\pi / 2, \mu=1$ and $\nu=-\frac{1}{2}$

$$
\int_{0}^{\pi / 2} J_{1}\left(2 k_{a} \cos \varphi\right) \cos ^{2} \varphi d \varphi=\frac{\Gamma\left(\frac{1}{2}\right) J_{3 / 2}(2 h a)}{\sqrt{2 k a} \sqrt{2}}
$$

$(A-10)$

and so

$$
\int_{0}^{\pi / 2} J_{1}(2 k a \cos \theta) \cos ^{2} \varphi d \varphi=j_{1}(2 k a)
$$

Therefore,

$(A-12)$

$$
T=-\frac{1}{2} \dot{j}_{1}(2 k a)
$$




\section{APPENDIX B}

FOURIER TRANSFORMS AND COHTOURS OF INTEGRATION

Using the Fourler Integral theorem (9), a

finite wave train or pulse may be represented mathemat1cally as

$$
f(t)=\frac{1}{2 \pi} \int_{-\infty}^{+\infty} e^{i \omega t} d \omega \int_{-\infty}^{+\infty} S\left(t^{\prime}\right) e^{-i \omega t^{\prime}} d t^{\prime}
$$

The quantity

$$
g(\omega)=\frac{1}{\sqrt{2 \pi}} \int_{-\infty}^{+\infty} S(t) e^{-i \omega t} d t
$$

is called the Fourier transform of $f(t)$.

1. Exponential Pulse:

For the exponential pulse, the acoustical disturbance is given by

$s(t)=e^{-\sigma t}$

$s(t)=0$

$$
t>0
$$$$
t<0
$$

The Fourier transform of this pulse is

$$
g(\omega)=\frac{-i}{\sqrt{2 \pi}(\omega-i \sigma)}
$$


42

2. Sinusoidal Pulse:

The acoustical disturbance for this type of pulse is

$S(t)=\sin \omega \frac{t}{i}=\sin \omega_{0} t \quad-\frac{T}{2}<t<\frac{T}{2}$

$(B-5)$

$$
S(t)=0
$$

$$
t>\frac{T}{2}, t<-\frac{T}{2}
$$

where

$$
\frac{T}{2}=2 n \pi, \quad \frac{\omega}{\tau}=\omega_{0}
$$

The Fourier transform becomes

$$
g(\omega)=\frac{-2 i \omega_{0} \sin \omega \frac{T}{2}}{\sqrt{2 \pi}\left(\omega-\omega_{0}\right)\left(\omega+\omega_{0}\right)}
$$

3. Note on Contour Integration:

The first integral in Equation (4.5) w111 be calculated as an example to demonstrate the contours used for integration in Chapter IV.

$(B-7)$

$$
I_{1}=\int_{-\infty}^{+\infty} \frac{e^{i \omega\left(t^{\prime}+\frac{2 a}{c}\right)} d \omega}{\omega(\omega-i \sigma)}
$$


The following contour in the complex $w$

plane has been chosen:

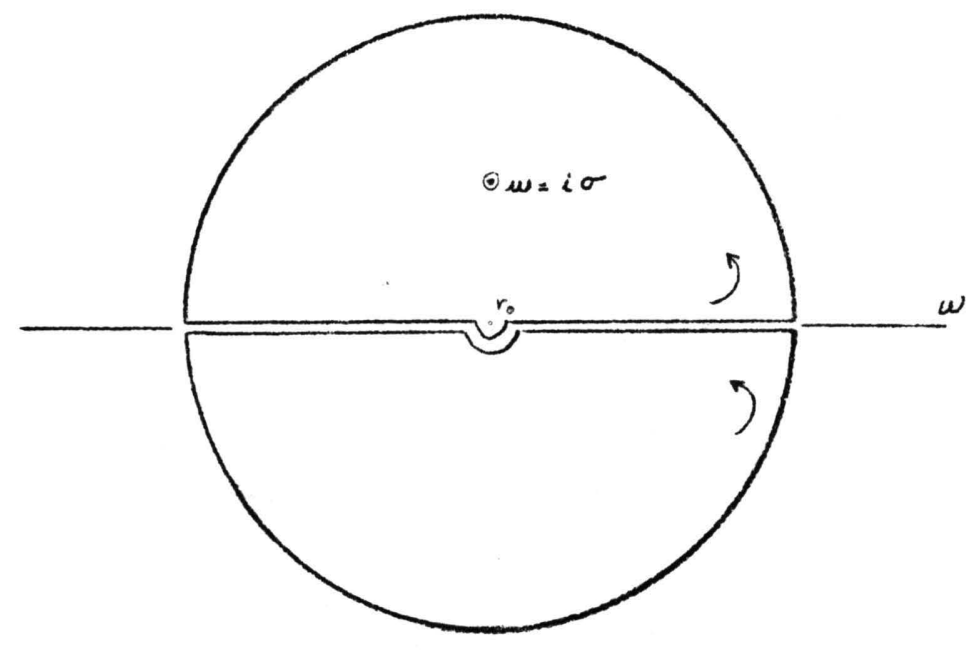

By observing the term $e^{i \omega\left(t^{\prime}+\frac{2 a}{c}\right)}$ it is evident that for $t^{\prime}+\frac{2 a}{c}>0$, the upper contour must be used and for $t^{\prime}+\frac{2 a}{c}<0$ the bottom contour must be used, since the function is singular at $w=i \infty$ for $t^{\prime}+\frac{2 a}{c}<0$ and at $w=-i \infty$ for $t^{\prime}+\frac{2 a}{c}>0$. This ensures that the value of the integral along the semicircle will vanish in the limit as the radius of the semiclrcle becomes infinite. The poles on the real axis may be included in elther contour but in this thesis they are always included in the upper contour.

From the theory of complex integration (10)

$$
\int_{C} f(z) d z=2 \pi i \sum \text { Residues }
$$


For $I_{1}$, Equation $(B-8)$ ylelds for $t^{\prime}+\frac{2 a}{c}>0$

$\int_{-\infty}^{+\infty} \frac{e^{i \omega\left(t^{\prime}+\frac{2 q}{e}\right)} d \omega}{\omega(\omega-i \sigma)}=2 \pi i\{$ Residue $(\omega=0)+$ Residue $(\omega=i \sigma)\}$

$$
-\int_{i}^{2 \pi} \frac{e^{i r_{0} e^{i \varphi}\left(t^{\prime}+\frac{2 a}{c}\right)} i r_{0} e^{i \varphi} d \varphi}{r_{0} e^{i \varphi}\left(r_{0} e^{i \varphi}-i \sigma\right)}
$$

where $\omega=r_{0} e^{i \phi}$ around the pole at the origin. Thus,

$$
\begin{aligned}
& I_{1}=\int_{-\infty}^{+\infty} \frac{e^{i \omega\left(t^{\prime}+\frac{2 a}{c}\right)} d \omega}{\omega(\omega-i \sigma)}=2 \pi i\left.\frac{1}{-i \sigma}+\frac{e^{-\sigma\left(t^{\prime}+\frac{2 a}{c}\right)}}{i \sigma}\right\} \quad(B-10) \\
&+\int_{\pi}^{2 \pi} \frac{d \varphi}{\sigma} \\
& I_{1}=\frac{2 \pi}{\sigma}\left\{e^{-\sigma\left(t^{\prime}+\frac{2 a}{c}\right)}-\frac{1}{2}\right\} \quad t^{\prime}+\frac{2 a}{c}>0
\end{aligned}
$$

and it clearly has the value

$$
I_{1}=\frac{\pi}{\sigma}
$$




\section{APPENDIX 0}

THE BORN APPROXIMATION FOR SPHERICAI SCATTERING

Since the acoustical properties of the sphere are very similar to those of the surrounding medium, the sphere may be considered a perturbation in an otherwise unbounded medium and the Born approximation may be used to calculate an approximate solution to the wave equation.

For the infinite medium, the scaler potential satisfies the three dimensional wave equation

$$
\nabla^{2} \phi+k_{1}^{2} \phi=0
$$

where $k$ is the wave number in that medium. When the sphere is introduced into the medium, the potential in the vicinity of the sphere changes slightly and the wave equation may be written

$$
\nabla^{2} \Phi_{1}+\left(k_{1}^{2}+\epsilon_{0}\right) \Phi_{1}=0
$$

where

$$
\epsilon_{0}=h_{2}^{2}-k_{1}^{2}
$$

and

$$
\frac{\epsilon_{0}}{h^{2}} \ll 1
$$

This problem is one which may well be approximated by the method due to Born for which an excellent presentation 
1s given by Schiff (7). Letting

$$
\phi_{1}=\phi_{i}+\phi_{s}=e^{-i k_{1} r \cos \theta}+\varphi_{s}
$$

and neglecting the term $\epsilon_{0} \Phi_{s}$, Equation $(C-1)$ becomes

$$
\nabla^{2} Q_{s}+k^{2} \varphi_{s}=-\epsilon_{0} e^{-i k r \cos \theta}
$$

The solution to this inhomogeneous equation is (7)

$$
\varphi_{s}=\frac{\epsilon_{0}}{4 \pi} \int_{0}^{a} \int_{0}^{\pi} \int_{0}^{2 \pi} d \phi_{0} r_{0}^{2} d r_{0} \operatorname{Sin} \theta_{0} d \theta_{0} \frac{e^{-i h\left\{\left|\vec{r}-\vec{r}_{0}\right|+r_{0} \cos \theta\right\}}}{\left|\vec{r}-\vec{r}_{0}\right|}
$$

The diagram defines all distances and angles.

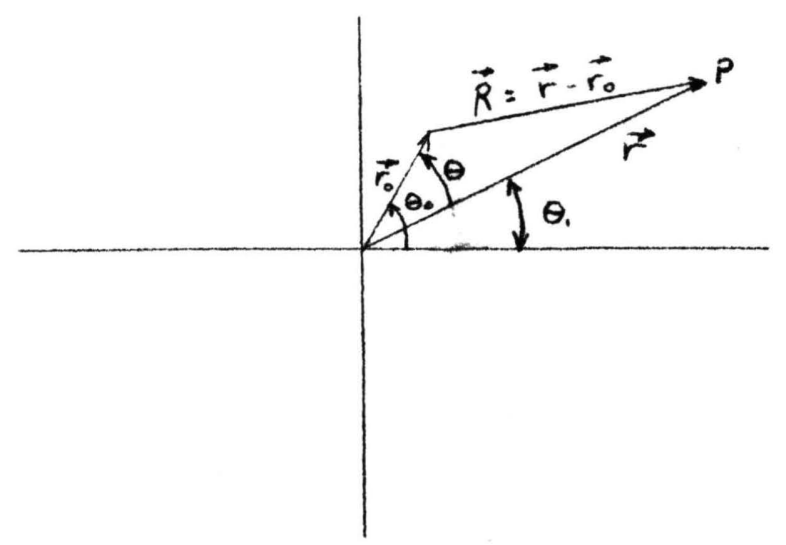

The distance $R=\left|\vec{r}-\vec{r}_{0}\right|=r-r_{0} \cos \theta$ and for large $\vec{r}$

$$
\begin{aligned}
& \left|\vec{r}-\vec{r}_{0}\right| \sim r \quad \text { For back-scattering } \theta_{1}=\pi \text { and } \\
& \theta=\theta_{0}-\pi \text {, and } \\
& \Phi_{s}=-\frac{e^{-i k r}}{2 k r} \epsilon_{0} \int_{0}^{a} \int_{0}^{\pi} r_{0}^{2} d r_{0} \operatorname{Sin} \theta_{0} e^{-2 i k r_{0} \cos \theta_{0}} d \theta_{0}
\end{aligned}
$$


Upon evaluating the integral, the Born approximation for the scattered field is

$$
\Phi_{s}=-\frac{e^{-i k r}}{2 k r} \omega^{2}(\epsilon-\delta)\left\{\frac{1}{4 k^{2}} \sin 2 k a-\frac{a}{k} \cos 2 k a\right\}
$$

This expression for $Q_{s}$ is essentlally the same as found by Pekeris (II)。 The integration may be extended easily to general angles of scattering. 


\section{BIBLIOGRAPHY}

I. Rayleigh, J. W. S. Baron, the Theory of Sound, 2, 282-284 (Dover Publications, New York, 1945).

2. Morse, P. M., Vibration and Sound (McGraw-Hill Book Company, Hew York, 1948).

3. Faran, J. J., "Sound Scattering by Solld Cylinders and Spheres," J.A.S.A. 23, 405 (1951).

4. Anderson, V. C., "Sound Scattering From a Fluid Sphere," J.A.S.A. 22, 426 (1951).

5. Ewing, W., Jardetzky, W. and Press, F., Elastic Waves in Lavered Media, Chapter I (MoGrawHill Book Company, Hew York, 1957).

6. Rayle1gh, J. W. S. Baron, The Theory of Sound, 2, 261 I (Dover Publications, New York, 1945 ).

7. Schiff, I. I., Quantum Mechanies, pp。161-171 (McGraw-HIII Book Company, New York, 1955).

8. Magnus, $W_{.}$, and obberhettinger, F., Formulas and Theorems for the special Functions of Mathematical physics Chelsea Publishing Co., NeW York, 1949).

9. Church1ll, R. V., Fourler Series and Boundary Value Problems, p. 89 McGraw-HIII Boak Company, New York, 1941).

10. Church111, R. V., Complex Varlables and Applications, p. 155 (MCGraw-HIII Book Co., New York, $1960)$.

11. Pekeris, C. I., "Note on the Scattering of Radiation in an Inhomogeneous Media," Phys. Rev. 11, 268 (1947). 


\section{ABSTRACT OF THESIS \\ ACOUSTIC SCATTERING BY FLUID SPHERES}

The problem of scattering of acoustic waves and pulses by an elastic sphere embedded in an infinite elast1c medium is investigated for the case where the two media are very similar acoustically. This physical situation allows functions with arguments involving the acoustic parameters inside the sphere to be expanded in a Taylor series involving the acoust1c parameters outside the sphere. Using only the first order terms in this expansion, the solution for plane wave conditions in the back-scattered direction is much simpler than the exact solution. This allows the solutions for the scattering of acoustic pulses to be calculated.

The steady state solutions are compared with those obtained using the Born approximation, and are found to differ only in the algebralc sign of the difference in density of the two media; although they agree wth the results obtained by Rayleigh in the proper limit. It is also found that the Born approximation differs from the results obtalned by Ravlelgh, again only in the algebraic sign of the difference in 
density of the two media.

Farlan G. Frey

Phys1cs Department

Colorado State University

rarch, 1962 